\title{
A Supply Chain Information System of the Supply-Hub Based on Blockchain
}

\author{
Junyi Guo \\ Guangxi University of Science and Technology, \\ No.268 Donghuan Road Liuzhou, Guangxi, China
}

\author{
Zhiping $\mathrm{Lu}$ \\ Guangxi University of Science and Technology, \\ No.268 Donghuan Road Liuzhou, Guangxi, China
}

\begin{abstract}
In view of that information asymmetry and information silos in the operation course of the supply chain logistics centre,the safety share of supply chain information is realized by using the Blockchain technology in combination with the information platform of supply chain logistics.By building a supply chain logistics information platform based on Blockchain technology, and describing and summarizing the technical structure of the platform,the current supply chain information sharing problems are solved and the application scenarios of Blockchain technology in the supply chain field are broadened.
\end{abstract}

Keywords-Blockchain; supply chain logistics information platform; supply chain coordination; Supply-Hub

\section{INTRODUCTION}

With the increase of social emergencies, the supply of manufacturing industry is unstable or even interrupted,which directly affects the suspension of production and the loss of core competitiveness. Therefore, the community began to pay attention to the cooperation between the supply chain and suppliers. The supply center supply logistics operation mode has been widely used in practice for its advantages of real-time distribution and cost reduction. However,in the face of the problems of many suppliers supplying and realizing JIT distribution requirements, the supply chain operation mode of the supply chain center puts higher requirements on information sharing.In addition, excessive information leakage and high system cost are also urgent problems to be solved in the operation mode of the Supply-Hub logistics.It is imperative to apply new technologies to achieve high sharing of information in the supply logistics operation mode of the Supply-Hub.

The decentralization of technologies such as Blockchain technology, cryptography technology,consensus mechanism, and smart contract is expected to solve the problem of information islands existing in current supply chain management[2].The pain point of the problem is the one-way circulation of information. The focus of this paper is to build a supply chain logistics information platform based on Blockchain technology,analyze the technical architecture of the platform, and analyze and discuss the advantages of the platform.

\section{OVERVIEW OF SUPPly CENTER SUPPly LOGISTICS}

Supply-Hubs are also known as VMI Hubs, Vendor Hubs, Material Hubs (centers), and Assembly Centers.Currently, Supply-Hub does not have a uniform definition.In a narrow sense, a supply center can be understood as a service integration facility located near an assembly manufacturing facility. According to the agreement,when the material is consumed, it is also called a collection center.From a broad perspective, the distribution links of distribution centers in supply chains and distribution centers are collectively referred to as "collection centers," which are coordinating organization suppliers and manufacturing assembly manufacturers between raw materials or components,and are primarily responsible for the entire supply chain system [4].

The main function of the supply chain hub is to become a component integration supplier and logistics service provider in the supply chain, to promote the coordinated production and coordinated supply of component suppliers and core manufacturers, while reducing costs and improving response speed. One of the effective modes of cooperative operation. In the Supply-Hub operation mode,the integration of inventory, transportation information and replenishment information of multiple suppliers requires the use of demand information to drive the production of manufacturers and suppliers. Under the supply chain hub operation mode,advanced information technology is urgently needed to promote information sharing while promoting information sharing,thereby realizing the stability and reliability of the supply chain system.

\section{SUPPLY CHAIN LOGISTICS INFORMATION PLATFORM BASED ON BLOCKCHAIN}

Blockchain is a new computational model that has emerged in recent years.Since its establishment,it has received wide attention from all walks of life.At present,the definition of Blockchain has not been unified. Many scholars have discussed the definition of Blockchain extensively and narrowly.Yong Yuan believes that,in a narrow sense,Blockchain is a nontamperable,unforgettable discretization that combines data blocks into specific data structures in a cryptographic manner [5].China's Ministry of Industry and Information Technology and the Ministry of Information Technology's "White Paper on China's Blockchain Technology and Application Development" defines the Blockchain in a broad sense,that is, Blockchain is a computer-based distributed data storage, point-

Fund: National Social Science Fund Project of China(18XGL006) 
to-point transmission and Encryption Algorithm.Application examples of new technologies,such as consensus mechanisms. On the basis of previous studies,this paper considers that the Blockchain is a chained database composed of blocks. The encryption technology ensures that the distributed distributed ledger technology has high credibility.

\section{THE APPLICATION OF BLOCKCHAIN IN THE FIELD OF SUPPLY CHAIN INFORMATION PLATFORM}

At present,the research on Block chain supply chain mainly focuses on supply chain finance,supply chain information, and supply chain application model.From the research information of the supply chain, the previous research mainly focused on the construction of logistics information platform,traceability and anti-counterfeiting of supply chain information, and information security certification.At the same time,the Blockchain is studied at a more detailed level,such as supply chain supply chain logistics. The study of information is still a blank.

\section{THE SUPPLY CHAIN LOGISTICS INFORMATION PLATFORM PARTICIPATES IN THE MAIN BODY}

The main body of the manufacturer alliance.Under the Blockchain,the manufacturer's inventory information,demand planning, production progress information,and upstream and downstream transaction information are packaged,distributed, and recorded on the platform. The digital signature technology of Blockchains prevents information from being tampered with. Each component supplier can view relevant information at any time according to the information sharing agreement to determine the quantity of supply and delivery date.The intelligent contract technology of the Blockchain realizes the automatic transfer of transaction funds and the automatic execution of claim contracts, which simplifies the transaction process and reduces transaction costs.

\section{A. Supplier alliance}

Information sharing between component suppliers is an important condition for multiple suppliers to meet the needs of downstream manufacturers quickly, accurately, and at low cost. Under the Blockchain, product structure information,inventory information,transportation information, parts shortage information, and arrival time information between multiple suppliers can be linked to the supply chain information platform. The member of the supplier alliance obtains relevant shared information based on the private key and the public key. Blockchain's smart contract technology automates transaction management, default compensation, and remediation management.

\section{B. Third party logistics operating entity}

The main transportation information,the delivery and storage information of the goods, and the distribution information owned by the third-party logistics operation entity can be shared by the manufacturer and the supply chain alliance entity on the platform. The information traceability function of the Blockchain can control the quality of the goods.

\section{Blockchain repair company}

Although supply chain information platform is built by supply chain alliance,it needs technical support,physical facilities,system construction and daily maintenance from professional technical companies such as Google [1].

\section{The main body of the manufacturer alliance}

Under the Blockchain,the manufacturer's inventory information, demand planning, production schedule information, upstream and downstream transaction information are packaged,distributed,and recorded on the platform. The digital signature technology of Blockchains prevents information from being tampered with.Each component supplier can view relevant information at any time according to the information sharing agreement to determine the quantity of

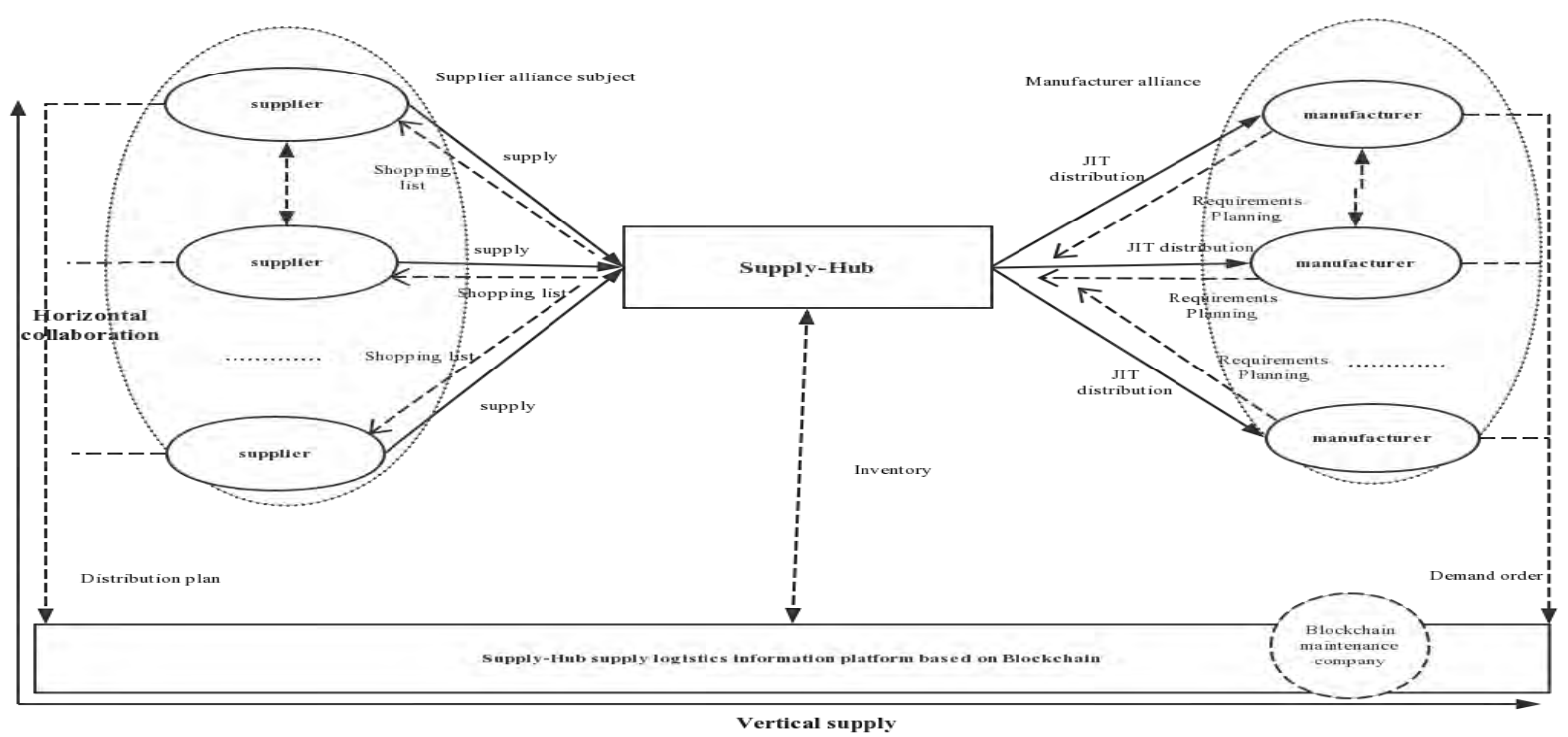

Fig. 1 Conceptual model of Supply-Hub supply chain logistics information platform based on Blockchain 
supply and delivery date. The intelligent contract technology of the Blockchain realizes the automatic transfer of transaction funds and the automatic execution of claim contracts, which simplifies the transaction process and reduces transaction costs.

\section{SUPPLY CHAIN LOGISTICS INFORMATION PLATFORM TECHNOLOGY STRUCTURE}

The Blockchain system consists of a data layer,a network layer,a consensus layer, an incentive layer,a contract layer,and an application layer.The formation platform based on Blockchain in proposed in this paper mainly includes three modules,namely the application module of the application layer, including the core module of the contract layer,the consensus layer and the application layer,and the data layer.

\section{A. Data layer}

The data layer of the Blockchain encapsulates the underlying data blocks and related data encryption and time stamping techniques,effectively ensuring the security of information acquisition.

\section{B. Contract level}

The contract layer consists of various script code, algorithmic mechanisms, and smart contracts. Among them, the smart contract is the core of the contract layer,realized by the contract code embedded in the Blockchain.Participants prepare the contract content and trigger mechanism in advance and embed the system in code form. Once the trigger condition is met, the contract is automatically executed.

\section{Application layer}

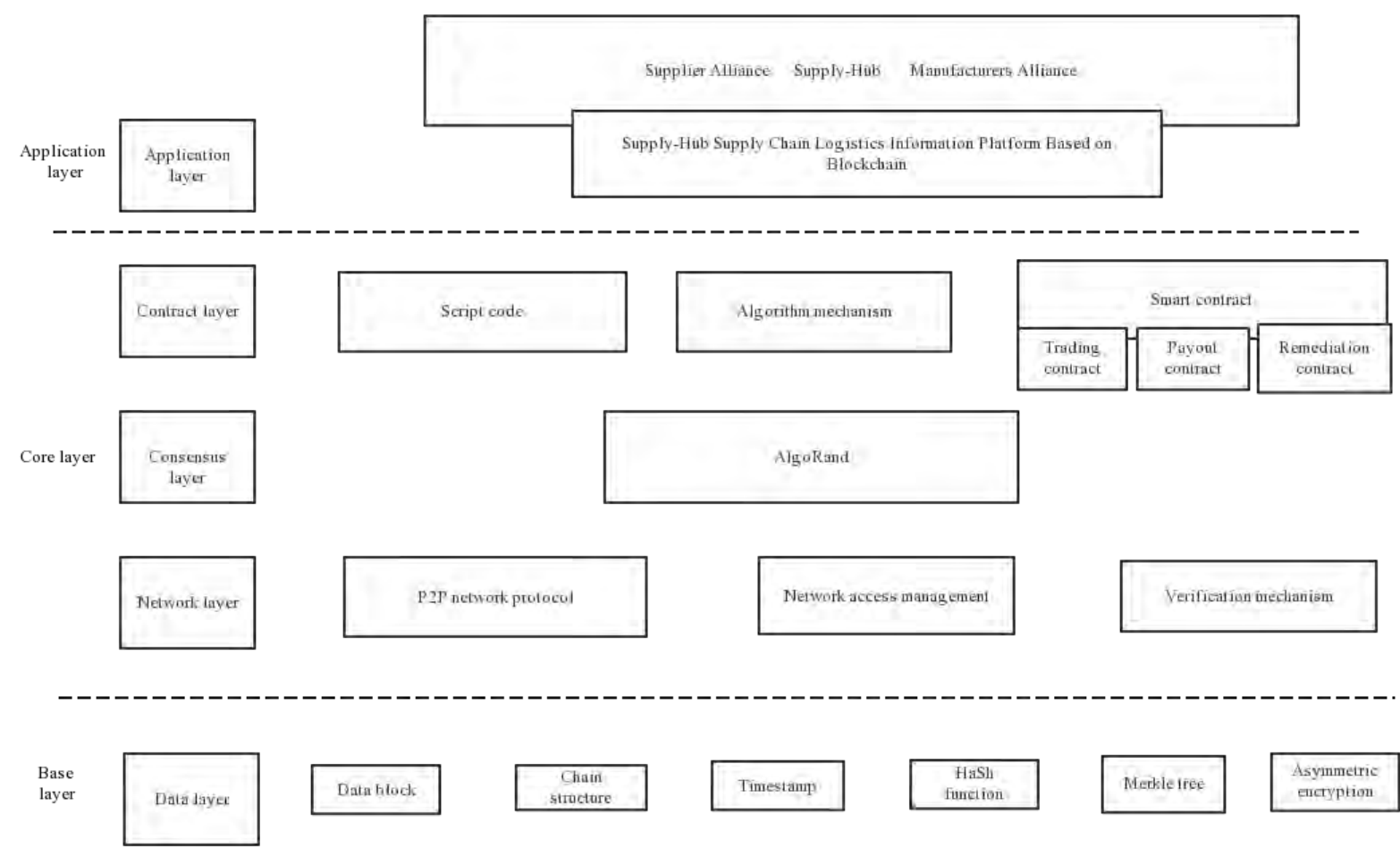

Fig. 2 Technical structure of Supply-Hub supply chain logistics information platform based on Blockchain

The application layer encapsulates the application scenario of the supply chain, and is the medium for the supplier alliance main body,the Supply-Hub, and the manufacturer alliance main body to interact with the information platform. The information platform records the entire process of logistics,business processes, and capital flows between suppliers and supplier alliance entities, Supply-Hubs, and manufacturer alliances, and achieves an upgrade of the value chain.

\section{Consensus layer}

How to effectively reach consensus in distributed systems is an important research topic in distributed computing. One of the core strengths of Blockchain technology is the ability to efficiently agree on the validity of block data in a decentralized system with highly decentralized decision making. So far, there are 32 important consistency algorithms in the Blockchain, including POW consistency algorithm,DPOS consistency algorithm,Paxos and Raft consistency algorithm, and hybrid class consistency algorithm. The supply chain logistics information platform of uses AlgoRand fast Byzantine fault-tolerant algorithm. The algorithm uses the password lottery technique to select the verifier and leader of the negotiation process, and agrees on the new block through the $\mathrm{BA} *$ Byzantine fault-tolerant protocol designed by the algorithm. AlgoRand requires only a minimum amount of computation and few branches, and is considered to be A truly democratic and efficient distributed ledger consistency technology. 


\section{ADVANTAGES OF SUPPLY CHAIN LOGISTICS INFORMATION PLATFORM BASED ON BLOCKCHAIN}

The current supply chain information system is a centralized system.Businesses line up and information flows in one direction. Distrust between members of the supply chain can affect the extent of information sharing and the quality of information.Blockchain has a high degree of autonomy, distributed equivalence, decentralization, traceability, collective maintenance and irreparable modification, which provides new ideas and directions for solving the pain points of current supply chain information systems.

\section{A. Reduce transaction costs}

The digital signature and encryption technology of the Blockchain makes it possible to reduce transaction costs in the automotive parts supply logistics operation model.Both sides of the model trade directly on the Blockchain,real-time viewing and real-time clearing of secure transactions, thereby eliminating intermediary transactions and reducing transaction costs.

\section{B. Share information securely}

The Supply-Hub supply logistics operation mode needs the support of information technology to realize the synchronous operation and information coordination of supply logistics. Under the Blockchain concept, chronological information blocks are collectively recorded by multiple nodes. This feature allows information to be shared securely throughout the system.

\section{Establish a reliable trust mechanism}

The premise of supply chain coordination is to establish a trust relationship. Transactions between supply chain entities are a competitive partnership.At the same time, in order to mutual trust, mutual support, and mutual recognition, a close trust relationship must be established. The Blockchain uses the smart contract to establish a credit transaction integrity mechanism that covers the entire chain, the entire process, and the entire employee.It uses a consensus mechanism and digital encryption technology to create a trusted system that does not require a third party to decentralize.

\section{Visual information}

The decentralization and group maintenance of Blockchains have made the problem of information silos and information one-way circulation in the process of information interaction increasingly prominent.In addition, through the electronic chip,the platform can record the production information of all members,including raw material procurement,product manufacturing, distribution,distribution and other information,to achieve product traceability,and strengthen product supervision.

\section{CONCLUSION}

In order to solve the two-dimensional collaborative supply base of information sharing,this paper combines the core advantages of Blockchain,and proposes the idea of applying Blockchain technology to supply logistics operation mode in the supply base,and builds logistics information platform based on Blockchain.The analysis shows that the information platform can solve the problem of low information sharing and excessive information leakage in the supply chain through the Blockchain. However,the system cost is too high,the Blockchain itself has technical problems, and the resource consumption is large, which will be the challenge for the future supply chain logistics information platform [6].

\section{ACKNOWLEDGMENT}

This paper was financially supported by National Social Science Fund Project of China (Project number:18XGL006)

\section{REFERENCES}

[1] Yang Huiqin, Sun Lei, Zhao Xichao. Construction of mutual trust and win-win supply chain information platform based on Blockchain[J]. Science \& Technology Progress and Policy,2018,35(05):21-31.(In Chinese)

[2] Zhang Xiaheng. Optimization of Supply Chain Management Mode Based on Blockchain [J]. China Circulation Economy, 2018,32(08):4250.(In Chinese)

[3] Ministry of Industry and Information Technology. White Paper on China's Blockchain Technology and Application Development (2016) [EB/OL].(2016-10-21)[2018-02-13].http://mt.sohu. Com/20161021/n470943606.Shtml.(In Chinese)

[4] LI Guo, MA Shihua, GONG Fengmei, WANG Zhaohua. Summary and Prospect of Research on Supply Logistics Collaborative Operation Based on Supply-Hub[J]. Journal of Mechanical Engineering, 2011, 47(20): 23-33.(In Chinese)

[5] YUAN Yong, WANG Feiyue. Current Status and Prospects of Blockchain Development[J]. Acta Automatica Sinica,2016(4):481494.(In Chinese)

[6] Swan M. Blockchain: Blueprint for a New Economy[M]// Blockchain : blueprint for a new economy. 2015. 